\title{
Inverse and Ill-Posed problems for nonlinear PDE: applications to life and social sciences
}

\author{
M. Shishlenin ${ }^{1,2,3 *}$, D. Lukyanenko ${ }^{4}$ \\ ${ }^{1}$ Institute of Computational Mathematics and Mathematical Geophysics SB RAS, Novosibirsk, Russia \\ ${ }^{2}$ Sobolev Institute of Mathematics SB RAS, Novosibirsk, Russia \\ ${ }^{3}$ Novosibirsk State University, Novosibirsk, Russia \\ ${ }^{4}$ Moscow State University, Moscow, Russia \\ *e-mail:mshishlenin@ngs.ru
}

Key words: inverse and ill-posed problems, Life and social sciences, numerical methods, control problems

Motivation and Aim: In mathematical models of life and social sciences, financial mathematics inverse and ill-posed problems are investigated for nonlinear convectiondiffusion-reaction $\mathrm{u}_{\mathrm{t}}+(\mathrm{v}, \nabla \mathrm{u})-\tilde{\mathrm{N}}(\mathrm{k} \nabla \mathrm{u})=\mathrm{A}(\mathrm{q}, \mathrm{u})$, where right-hand side $\mathrm{A}$ is nonlinear in $q$ and $u$, respectively [1]. We consider the coefficient inverse problems of recovering $\mathrm{v}, \mathrm{k}$ and $\mathrm{q}$ by nonlocal data (integral over the domain given in the discrete time) [2]. This data characterizes a certain reference group (social survey), medicine (drag absorption) or financial market. Desired coefficients can characterize such important characteristics as labor-power ratio, labor productivity, consumption, interpersonal interactions, etc. Also we consider the control problem: how to find the source or initial function to obtain desired statement (or people behavior) in a final fixed time.

Methods and Algorithms: Due to extremely nonlinearity we reduce the inverse and illposed problem solution to the optimization problem. We apply the gradient method of minimizing the cost functional. A gradient of the functionals were obtained by solving the corresponding conjugate problems $[1,2]$.

Conclusion: The examples of ill-posedness were constructed. We also mention the theoretical and numerical results for considered problems. Numerical results are presented.

Acknowledgements: Supported by the RFBR (18-01-00865,18-41-540017, 17-51-540004, 16-01-00755).

\section{References}

1. Lukyanenko D.V., Shishlenin M.A., Volkov V.T. (2018) Solving of the coefficient inverse problems for a nonlinear singularly perturbed reaction-diffusion-advection equation with the final time data. Communications in Nonlinear Science and Numerical Simulation. 54:1339-1351.

2. Kabanikhin S.I., Shishlenin M.A. (2018) Recovering a Time-Dependent Diffusion Coefficient from Nonlocal Data. Numerical Analysis and Applications. 11(1):38-44. 